\title{
Determination of Maximum Power Transfer Conditions of Bimorph Piezoelectric Energy Harvesters
}

\author{
Mahmoud Al Ahmad ${ }^{1}$, Amro M. Elshurafa ${ }^{2}$, Khaled N. Salama ${ }^{2}$ and H. N. Alshareef ${ }^{*}$ \\ ${ }^{1}$ Material Sciences and Engineering, Physical Sciences and Engineering Division, King Abdullah \\ University of Science \& Technology (KAUST), Thuwal, Saudi Arabia, 23955-6900. \\ ${ }^{2}$ Electrical Engineering, Physical Sciences and Engineering Division, King Abdullah University of \\ Science \& Technology (KAUST), Thuwal, Saudi Arabia, 23955-6900.
}

*Corresponding author: husam.alshareef@kaust.edu.sa

\begin{abstract}
In this paper, a method to find the maximum power transfer conditions in bimorph piezoelectric-based harvesters is proposed. Explicitly, we derive a closed form expression that relates the load resistance to the mechanical parameters describing the bimorph based on the electromechanical, single degree of freedom, analogy. Further, by taking into account the intrinsic capacitance of the piezoelectric harvester, a more descriptive expression of the resonant frequency in piezoelectric bimorphs was derived. In interest of impartiality, we apply the proposed philosophy on previously published experimental results and compare it with other reported hypotheses. It was found that the proposed method was able to predict the actual optimum load resistance more accurately than other methods reported in the literature.
\end{abstract}

\section{Introduction}

Given their relatively low efficiency, the maximum power transfer is of extreme importance in MEMS piezoelectric bimorph harvesters. It is well known in circuit theory that maximum power transfer is achieved by impedance matching, i.e. when the load impedance equals the complex conjugate of the source impedance [1]. In piezoelectric scavengers however, finding optimum conditions is difficult because the mechanical domain (i.e. the stress and strain induced in the scavenger due to vibrations) is involved and is interacting with the external electrical load.

As such, different methods have been sought to model this power transfer and to find the optimum power transfer conditions. In [2] for example, the authors hypothesize that the maximum power is transferred to the electrical load when the resistance of the electrical load is equal to the resistance of the harvester, as with purely electrical networks. This latter assumption however, does not account for the mechanical damping. The authors in [3] claimed that the power transfer is optimized when mechanical damping is equal to electrical damping, while Williams et al. [4] claimed that the value of the electrical resistance of the load should be equal to the value of the virtual electrical equivalent resistance representing the mechanical damping. Further, the authors in [5] assume that, for maximum power transfer, the electrical load resistance should equal the sum of the harvester electrical internal resistance and the electrical analogy of the mechanical damping coefficient. The scientific community has acknowledged all these latter techniques, but results still need further analysis and validation.

In this work, the single degree of freedom circuit model for piezoelectric generators [6], based on the equivalent circuit representation of electromechanical analogy, is used to derive the maximum power transfer conditions in piezoelectric transducers. In order to verify the rationale and the analytical model proposed, experimental results based on published data are shown and indeed agree very well with the proposed theoretical analysis. 


\section{Theory}

Piezoelectric bimorphs constitute of two piezoelectric beams that are separated by a shim. The three latter layers are usually fixed on one side and left free at the other. When a mechanical load is applied to the bimorph, the strain induced in the piezoelectric material generates a voltage, which represents an energy conversion from the mechanical domain to the electrical domain; a transformer with a special turn ratio accounts to this conversion. Modeling piezoelectric bimorph harvesters using an electric circuit based on the electromechanical analogy is shown in figure 1 [6-9].

In figure 1, the left side of the network model represents the mechanical domain, where $\sigma_{\text {in }}$ models the input vibrations, $Z_{i n}$ models the input impedance of the network, $R_{b}$ models the mechanical damping, $L_{m}$ models the mass of the harvester, and $C_{k}$ models the inverse of the stiffness. At resonance, in which by definition the total impedance seen by the source is purely resistive, and using Kirchoff's voltage law, the mesh voltage relation on the primary side of the network in figure 1 is:

$$
\sigma_{\text {in }}=R_{b} \dot{S}+n V
$$

where $S$ is the strain, $n$ is the turn ratio of the transformer, and $V$ is the voltage, and the 'dot' represents the derivative. The voltage $V$ is given simply by:

$$
V=i R
$$

where $i$ is the current and $R$ is the load resistance. Therefore

$$
\sigma_{\text {in }}=R_{b} \dot{S}+n i R
$$

The current generated as a result of the mechanical stress evaluated at zero electric field is [8],

$$
i=a w l_{e} d_{31} c_{p} \dot{S}=\alpha \dot{S}
$$

where $a$ is a constant that is 1 or 2 depending on the wiring of the harvester, $w$ is the width of the piezoelectric material, $l_{e}$ is the length of the electrode in the piezoelectric harvester, $d_{31}$ is the piezoelectric strain coefficient, and $c_{p}$ is the Young's Modulus of the piezoelectric material, and $\alpha$ is the product of $a$, $w, l_{e}, d_{31}$, and $c_{p}$.

Hence, and after substituting equation (4) into (3),

$$
\sigma_{i n}=\left(R_{b}+n \alpha R\right) \dot{S}
$$

Equation (5) provides important insight regarding the transfer of impedances in electromechanical analogy networks. By relying on power system theory, the equivalent load resistance should be carried to the primary side and hold a new value of $n^{2} R$. However, equation (5) explicitly indicates that the load resistance will be carried to the primary side with a scaling factor equal to $n \alpha$. This finding is one of the important contributions of this communication.

Now, the optimum corresponding mechanical power delivered to the electrical domain, $P_{L}^{M}$, assuming maximum power transfer is

$$
P_{L}^{M}=\frac{n \alpha R}{\left(R_{b}+n \alpha R\right)^{2}} \sigma_{i n}^{2}
$$

The electrical power, $P_{L}^{E}$, delivered to the load $R$ can be computed as: 


$$
P_{L}^{E}=i_{R}^{2} R
$$

where $i_{R}$ is the current passing through the load, and it can be calculated using the current divider rule in terms of the applied mechanical strain rate as

$$
i_{R}=\frac{i}{1+j \omega C_{b} R}
$$

where $\omega$ is the radial frequency, $C_{b}$ is the capacitance of the piezoelectric bender, and $j$ is the complex number. Therefore,

$$
i_{R}=\frac{\alpha \dot{S}}{1+j \omega C_{b} R}
$$

Solving equation (5) for $\dot{S}$ and substituting the result into equation (9) yields,

$$
i_{R}=\frac{\alpha}{\left(1+j \omega C_{b} R\right)} \frac{\sigma_{\text {in }}}{\left(R_{b}+n \alpha R\right)}
$$

Consequently, the power delivered to the load now becomes

$$
P_{L}^{E}=\frac{\alpha^{2} R}{\left(1+j \omega C_{b} R\right)^{2}} \frac{\sigma_{i n}^{2}}{\left(R_{b}+n \alpha R\right)^{2}}
$$

For maximum power transfer, we take the derivative of equation (11) with respect to $R$ and equate it to zero. After solving, the optimum resistance, $R_{o p t}$, is found to be:

$$
R_{o p t}=\left\lfloor\frac{R_{b}}{\left.\llbracket n \alpha+j \omega C_{b}\left(R_{b}+2 n \alpha\right)\right]} \mid\right.
$$

and the maximum power is obtained by substituting the value of $R_{\text {opt }}$ as derived in equation (12) into equation (11).

Now we turn the attention to $R_{b}$, which represents the electrical analogy of the mechanical damping; $R_{b}$ can be expressed as follows:

$$
R_{b}=k_{1} k_{2} b_{m}
$$

where $b_{m}$ is the damping coefficient, $k_{1}$ is a constant that relates stress to force, and $k_{2}$ is a constant that relates strain to deflection. $k_{1}, k_{2}$ and $b_{m}$ are given by [8]:

$$
\begin{aligned}
& k_{1}=\frac{b\left(2 l_{b}+l_{m}-l_{e}\right)}{2 I} \\
& k_{2}=\frac{l_{b}^{2}\left(2 l_{b}+3 / 2 l_{m}\right)}{3 b\left(2 l_{b}+l_{m}-l_{e}\right)} \\
& b_{m}=2 m \xi \omega_{n}
\end{aligned}
$$

where $b$ is the distance between the center of the shim to the center of the piezoelectric material, $l_{b}$ the length between the fixed end and the proof mass, $l_{m}$ is the length of the mass, $m$ is the mass, $\xi$ is the unitless damping ratio, $I$ is the moment of inertia of the composite bimorph, and $\omega_{n}$ is the resonant frequency. Substituting (10), (11) and (11) in (9), yields: 


$$
R_{b}=\frac{l_{b}^{2} \xi \omega_{n} m\left(4 l_{b}+3 l_{m}\right)}{6 I}
$$

where $I$ is the effective moment of inertia of the composite bimorph.

\section{Resonant frequency derivation}

All the above analysis was assumed to have taken place at resonance. As such, it is extremely crucial to derive the resonance frequency with accuracy given that the electrical and mechanical domains are interacting.

To find the resonant frequency, $C_{b}$ should be transferred to the primary side using the factor $n \alpha$, and the input impedance is obtained as

$$
Z_{\text {in }}=R_{b}+j \omega L_{m}+\frac{1}{j \omega C_{k}}+\frac{n \alpha}{j \omega C_{b}}
$$

At resonance, the imaginary part of the impedance goes to zero. Hence, we write

$$
j \omega L_{m}-\frac{j}{\omega C_{k}}-\frac{j n \alpha}{\omega C_{b}}=0
$$

Finally, the resonant frequency is obtained as:

$$
\omega_{n}=\sqrt{\frac{1}{L_{m}}\left(\frac{1}{C_{k}}+\frac{n \alpha}{C_{b}}\right)}
$$

Note that if the capacitance $C_{b}$ was not present, then the resonant frequency expression reduces to the well known expression of $1 /\left(L_{m} C_{k}\right)^{0.5}$ for a typical series $R L C$ circuit. Indeed, this was the practice in previous publications. In other words, the intrinsic capacitance of $C_{b}$ was ignored. We can also rewrite equation (14) as:

$$
\omega_{n}=\sqrt{\frac{1}{L_{m} C_{k}}\left(1+\frac{n \alpha C_{k}}{C_{b}}\right)}
$$

and the term $n \alpha C_{k} / C_{b}$ governs the discrepancy between calculating the resonant frequency from the mechanical domain alone or calculating the resonant frequency by taking into account both the electrical and mechanical domains.

However, it was found that by assuming typical values for $n, \alpha, C_{k}$, and $C_{b}$, a difference of approximately $5 \mathrm{~Hz}$ exists between the resonant frequency calculated if $C_{b}$ was taken into account or not. Given that most piezoelectric scavengers rely on frequencies in the range of $50 \mathrm{~Hz}$ to $200 \mathrm{~Hz}$ and their bandwidth is small, a $5 \mathrm{~Hz}$ shift in the operating frequency from the resonant frequency could be detrimental to the performance of the harvesters. This finding is also particularly important for the choice of the proof mass, 
which is used to tune the resonant frequency of the harvester to match the frequency of the vibrations (which act as the excitation), to ensure maximum power transfer.

\section{Validation and discussion}

As stated earlier, the focus of this work is to find the best possible conditions ensuring maximum power transfer for piezoelectric bimorphs excited by vibrations. In interest of impartiality, we opt to chose previously published experimental results, and compare them to the method proposed here along with other philosophies as well. The reported results in $[10,11]$ have been chosen to validate the proposed model.

Figure 2 shows the measured output active power for the piezoelectric harvester at a resonant frequency of $95 \mathrm{~Hz}$ versus a range of load resistances $[10,11]$. All parameters and material properties used in the model implementation were reported in $[10,11]$; nonetheless, we include them in table 1 for convenience. As can be seen, the experimental data indicate that the maximum power is achieved at a load resistance of $80 \mathrm{k} \Omega$. The method proposed here was able to arrive at this resistance within the smallest range of error compared to other well-known methods.

Table 1: Parameters and material properties used in [10] and herein for the modeling.

\begin{tabular}{cc}
\hline Parameters or material property & Value \\
\hline$c_{p}$ & $62 \mathrm{GN} / \mathrm{m}^{2}$ \\
Young's Modulus of the Shim material $\left(c_{s h}\right)$ & $66 \mathrm{GN} / \mathrm{m}^{2}$ \\
$w$ & $3.2 \mathrm{~mm}$ \\
Dielectric constant $(\varepsilon)$ & 1800 \\
Piezoelectric material thickness $\left(t_{c}\right)$ & $0.134 \mathrm{~mm}$ \\
Shim thickness $\left(t_{s h}\right)$ & $0.132 \mathrm{~mm}$ \\
$l_{e}$ & $25.60 \mathrm{~mm}$ \\
$m$ & $0.5894 \mathrm{grams}$ \\
$\xi$ & 0.02 \\
Acceleration of gravity $(g)$ & $9.8 \mathrm{~m} / \mathrm{s}^{2}$ \\
$d_{31}$ & $-1.9 \mathrm{e}-10 \mathrm{~m} / \mathrm{V}$ \\
Piezoelectric coupling coefficient $\left(k_{31}\right)$ & 0.32 \\
\hline
\end{tabular}

Figure 2 was generated with the aid of table 2, where table 2 provides a list of the optimum resistance values that are predicted based on the assumptions of their respective authors. In [2], it was argued that the maximum power transfer would take place when the load resistance is equal to the harvester resistance. This argument is surely sensible, however the harvester resistance is hard to define (i.e. define it relative to the width of the beam, length of the beam, etc). Further, it has a fundamental drawback, which is ignoring the damping of the vibration. As such, this method was not able to find the optimum power of the case under study.

For the authors in [4], it was also sensible to hypothesize that the maximum power transfer would take place when the load resistance would equal the mechanical resistance (which represents mechanical damping) based on circuit theory. As shown in this paper, this concept is fundamentally correct, but its execution was lacking the correct impedance transformation as shown in section 2 . The results acquired through the methodology in [12], where an equivalent circuit at resonance representing the piezoelectric generator is used, was also logical but once again did not take into account the losses represented by $R_{b}$. 
Of all the reported assumptions, the method reported by the authors in $[6,8]$ was able to predict the maximum power conditions rather well. The argument was based on equating the electrical and mechanical damping ratios. By doing that, the coupling between the electrical and mechanical domains is taken into account effectively. The method we propose here takes into account the coupling between the two domains as well, and hence is able to predict the value of the optimum resistance.

Finally, it is important to note that the model presented in this paper is linear while the stress distribution in the bimorph is not; this will come at the expense of reduced accuracy. To account for the nonlinearity of the piezoelectric layer, a higher order circuit model must be used. Considering the simplicity of the introduced model, it is considered very effective in finding the optimal power conditions especially when the bending of the bimorph is not large, i.e. the nonlinearity in the stress gradients is not severe [13].

\section{Conclusion}

The maximum power transfer in a piezoelectric generators based on the electromechanical analogy is analyzed, and a rationale to find the optimum conditions was provided. It was found that reflecting impedance from one side of the electromechanical analogy circuit to the other does not follow the usual impedance reflection rules known in network theory; this was the primary source of error in previous publications that hindered finding the optimum resistance accurately. Further, a more descriptive expression for the resonant frequency in piezoelectric scavengers was derived; this was achieved by taking into account the intrinsic capacitance of the piezoelectric harvester. With the aid of the latter two important findings, closed from expressions for the power and optimum resistance were derived. After comparing the method proposed herein with other reported approaches in the literature, it was found that the proposed method provides an accurate and simple technique to find optimum power output conditions in piezoelectric bimorphs.

\section{References}

[1] R. Meadows, Electric network analysis: Burns \& Oates, 1972.

[2] M. El-Hami, et al., "Design and fabrication of a new vibration-based electromechanical power generator," Sensors and Actuators A: Physical, vol. 92, pp. 335-342, 2001.

[3] S. Roundy, P. Wright, and J. Rabaey, "A study of low level vibrations as a power source for wireless sensor nodes," Computer Communications, vol. 26, pp. 1131-1144, 2003.

[4] C. Williams, et al., "Development of an electromagnetic micro-generator," 2002, pp. 337-342.

[5] N. Stephen, "On energy harvesting from ambient vibration," Journal of Sound and Vibration, vol. 293, pp. 409-425, 2006.

[6] S. Beeby, M. Tudor, and N. White, "Energy harvesting vibration sources for microsystems applications," Measurement science and technology, vol. 17, p. R175, 2006.

[7] A. Flynn and S. Sanders, "Fundamental limits on energy transfer and circuit considerations for piezoelectric transformers," Power Electronics, IEEE Transactions on, vol. 17, pp. 8-14, 2002.

[8] S. Roundy and P. Wright, "A piezoelectric vibration based generator for wireless electronics," Smart Materials and Structures, vol. 13, p. 1131, 2004.

[9] H. A. C. Tilmans, "Equivalent circuit representation of electromechanical transducers: I. Lumped paramter systems," J. Micromech. Microeng., vol. 6, pp. 157-176, 1996.

[10] J. Ajitsaria, "Modeling and analyis of PZT micropower generator," PhD Dissertation, Mechanical Engineering Department, Auburn University, 2008.

[11] J. Ajitsaria, S. Choe, D. Shen, and D. Kim, "Modeling and analysis of a bimorph piezoelectric cantilever beam for voltage generation," Smart Materials and Structures, vol. 16, p. 447, 2007.

[12] S. Priya, "Modeling of electric energy harvesting using piezoelectric windmill," Applied Physics Letters, vol. 87, p. 184101, 2005.

[13] M. Al Ahmad, A. M. Elshurafa, K. N. Salama, and H. N. Alshareef, "Modeling of MEMS Piezoelectric Harvesters Using Electromagnetic and Power System Theories," Smart Materials and Structures, Vol. 20, No. 8, 085001 (6pp), 2011. 


\section{Figure Captions}

Figure 1 Circuit Model for a piezoelectric generator.

Figure 2 Measured output power versus a range of load resistances at $95 \mathrm{~Hz}$. The maximum power is achieved at a load resistance of $80 \mathrm{k} \Omega$. The method proposed here was able to arrive at this resistance within a small error. 


\section{Table Captions}

Table 1 Parameters and material properties used in [10] and herein for the modeling

Table 2 List of approaches reported for modeling power output of piezoelectric harvesters 